\title{
Industry 4.0: Models, Tools and Cyber-Physical Systems for Manufacturing
}

\author{
(Guest Editorial)
}

\author{
Goran D. Putnik \\ Department of Production and Systems Engineering, University of Minho, Portugal \\ ALGORITMI Research Centre, University of Minho, Portugal \\ Luis Gonzaga Martins Ferreira \\ School of Technologym Polytechnic Institute of Cávado and Ave, Portugal \\ ALGORITMI Research Centre, University of Minho, Portugal
}

This issue of yhe FME Transactions comprises 30 papers of which the first 19 papers address Industry 4.0 concept making this FME Transactions issue a Special Issue with the theme titled: "Industry 4.0: Models, Tools and Cyber-Physical Systems for Manufacturing". These 19 papers came from two sources. The first source was through invitation to the authors of selected papers presented at the international conferences "8th International Conference on Business Sustainability - BS 2018" and "7th International Conference on Virtual and Networked Organizations Emergent Technologies and Tools - ViNOrg'18', both held in November 07-09, 2018, in Povoa de Varzim, Portugal, to submit extended version of the papers, and the second source was invitation to the reknown authors for submission of their research results within the Special Issue's theme. All papers were evaluated independetly through double blind review and after taking into consideration of the reviewers' comments and recommendations, the revised versions were submitted and after final evluation the papers were accepted for inclusion in this Special Issue.

After these 19 papers, the present FME Transactions issue includes also 11 papers on other themes.

Concerning the main theme of this Special Issue, the Industry 4.0 (I40) represents a significant step in the processes transformation in practically every industry. Seen as the fourth relevant industrial revolution, from the steam engine appearance for mechanization in the first, the electrical energy and mass production in the second, the automatization with computers and robots in the third, to nowadays, where the smart concept emerges in autonomous decisions and cyber-physicalsystems based systems. The technology played a key role in all events of this timeline, and its rapid and continuous evolution gives opportunities and challenges, forcing the processes to continuously adapt and people to assimilate those transformations. The initial subsistence tools-based economy evolved towards nowadays digital and global context where digital technology and virtual collaboration sustains both the manufacturing processes and relations among people (social networks) and companies (virtual organizations).

Computers, Internet, Cloud Computing and Internet of Things are keys enabling support for this technology impact. I4.0 can be seen as the peak of opportunities (new processing capabilities and instruments) and consequent needs and challenges (new systems, processes and algorithms), where the integration and control of existing and new systems, including the active and effective human participation, looks required as never before. The Big Data for processing huge amount of unstructured data that arises from multiple new sources, together with the Artificial Intelligence (AI) and cognitive computing that reinforce the capacity to analyze, decide and learn on top of those datasets, and the increasingly performance computing on top of distributed computing systems, represents a concrete example of technology exploration and opportunities.

The products life cycle is increasingly short and dynamic, the mass production must be customeroriented and individualized production is required. The required quality and the tenue supply opportunity, represent preconditions for the expected dynamic reconfigurations that I4.0 will determine. Considering the dynamics of this scenario, a question for Industrial and Computational Engineering arises: could it be possible to completely or partially reconfigure a process (equipment, programs and persons), maintaining the same high quality of services for keeping the (new) customer satisfied?

The capacity to mitigate timely production errors or defaults, demands the capacity to tune or change initial settings or even reprogramming supporting systems. Cyber-Physical Systems are announced as the new concept for systems that should allow the efficient integration between physical and digital components, supporting its cooperation and collaboration, in any phase of the manufacturing process. However, there isn't yet a global and accepted definition. 
The continuous dynamic reconfiguration tends to optimize and deal with the expected uncertainty. Collaborative processes are essential, and the coanalysis or co-decision should be a need. Human cognitive capacity has a special job in all process, being the automation supported by machines and systems.

Although several other aspects deserve to be explored, the main propose of this special issue (SI) must be seen in two different perspectives: the former is to better understand the I4.0 concept and its real effects on manufacturing industry, inherent processes and stakeholders interaction; the other intends to expose the role played by technologies (mainly Information and Communications Technology (ICT)) in adhering to I4.0, its challenges and opportunities.

Industry 4.0 being an important movement that transforms ideas and concepts, the research structure of its concepts and dynamics, understanding the created value or existing gaps, dependencies, requirements or opportunities, can be formulated on five hierarchical levels:

- Description: definitions, terminology, elements, objects, relations;

- Models and Behaviour: models and functions, performance measures, invariants;

- Mechanisms of choice: management of implementtations, validations, testing;

- Methods and Tools: implementation instruments (hardware, equipment, architectures, strategies for implementation, design), development methodologies and platforms

- Epistemology: theorical fundamentals, education, how the knowledge is acquired and used, validity and coherence of knowledge in social context, phenomenology, critique (e.g. limits and sense), human and social dimension (e.g. value), and other epistemological issues.

The main focus and scientific contribution of the papers presented on this SI and these levels are mapped in the next table. Some papers were classified in more than one level.

\begin{tabular}{llc}
\hline Levels & Papers & $\begin{array}{l}\text { Number } \\
\text { of Papers }\end{array}$ \\
\hline $\begin{array}{l}\text { Description } \\
\text { Models and }\end{array}$ & {$[1]$} & 1 \\
$\begin{array}{l}\text { Behavior } \\
\text { Mechanisms of }\end{array}$ & {$[1][2][4][5][6][7][8]$} & 7 \\
choice & {$[9]$} & 1 \\
$\begin{array}{l}\text { Methods and tools } \\
\text { Epistemology }\end{array}$ & {$[10][11][12][13][14][15][16][17]$} & 8 \\
\hline
\end{tabular}

As easily seen, this SI has a dominant contribution to Models, Behavior and Tools. Follow a list of the main contribution of each paper and the interest in its lecture.

The first paper, "What is a Cyber-Physical System: definitions and models spectrum" by Goran D. Putnik, Luis Ferreira, Nuno Lopes and Zlata Putnk, identifies and characterizes Cyber-Physical Systems (CPS) definitions and models in a spectrum distribution, stressing its coherence, but still far from an intelligent and effective CPS (I-CPS). The fundamentals for such advanced control systems are presented, where double loop learning process allows its supporting algorithms reprogramming, required invariant for Industry 4.0.

The second paper, "Cyber-Physical Production System (CPPS) decision making duration time impact on manufacturing system performance" by Cátia Alves and Goran D. Putnik, explores the impact of Industry 4.0 on the manufacturing system control and management processes. The authors describe the variation of completion time of the jobs given at the input and the CPPS decision-making duration time, considering stable and dynamic environments.

The third paper, "Using Simulation to Model the Logistic Operations of a Company of the Cement Industry", by António AC Vieira, Hugo Veloso, Luís MS Dias, Guilherme AB Pereira, José A Oliveira, M Sameiro Carvalho and Manuel C Figueiredo, presents a discrete-event simulation model to study the logistics operations of weighing, loading and unloading of raw materials inherent to cement industry in the Industry 4.0 era transformations. The model focused on the companies' requirements for logistics flows and their impacts on their performance and was validated with simulation experiments of existing flow of vehicles. The obtained results reveal the maximum storage capacity and flow of trucks required to unload the raw materials.

The management of innovations in Industry 4.0 (I40) is, for sure, another challenge that requires special attention. The fourth paper "Adaptive identification of innovative production function of corporation", by Vladimir V. Tsyganov, explores this requirement in the I4.0 context of manufacturing industry transformation. The I4.0 requires continuous renewal and development to deal with the uncertainty, challenges and opportunities that arise from competing companies, technologies, equipment and processes. The authors describe an interesting formal description for an innovative production function that supports a cycle of development and implementation of innovations.

The fifth paper, "A Human Centred Hybrid MAS and meta-heuristics based system for simultaneously supporting scheduling and plant layout adjustment", by Filipe Alves, Maria Leonilde R. Varela, Ana Maria A. C. Rocha, Ana I. Pereira and Paulo Leitão, presents an approach to solve problems of job shop scheduling in production systems, in the expected Industry 4.0 context where continuous layout adjustments for dynamic and flexible responses are required. Genetic algorithms and particle swarm optimization algorithm are applied.

The sixth paper, "Alignment of cluster complexity at network systems", by A.K. Enaleev and V.V. Tsyganov, proposes methods for solving partitioning problems of large-scaled networks, using complexity index alignment. It describes the method of clustering management applied on large-scale transport networks, in this case, applied on the high-speed railway project "Moscow-Kazan". That model requires data clusters and 
existing dependencies identification and their complexity measured. Big data processes are used for data clusters extracting and analysis, and heuristics algorithms of partitioning and alignment are used for cluster complexity alignment, are described. A paper that gives insights for the relevance of Big Data on I4.0.

The seventh paper, "Dynamic distribution of assembly tasks in a collaborative workcell of humans and robots", by Dario Antonelli and Giulia Bruno, emphasizes one of the most relevant topics of Industry 4.0 , the collaboration. Indeed, never than before, machines, processes and persons need to collaborate. The authors explore the arising issues when machines (robots) and humans need to collaborate, leaving complex tasks to robots and freeing man for more cognitive tasks. The announced new working paradigm.

The eighth paper, "A Strategy for Human-Robot Collaboration in Taking Products Apart for Remanufacture", by Jun Huang, Duc Truong Pham,Yongjing Wang, Chunqian Ji, Wenjun Xu, Quan Liu and Zude Zhou, strengthens the subject of the seventh paper where human and machines should collaborate on remanufacturing operations to disassembly end-of-life products. The authors propose a strategy for such collaboration.

The ninth paper, "Internet of Things Evolution: A European Perspective”, by Jorge Oliveira e Sá, José Luís Pereira and João Cacho, describes the evolution of the European Strategy for Internet of Things during the last decade. The research was based on the IoT technology development, results from related research clusters and applied projects. According to the authors, this quantitative analysis prepared the future one that should be qualitative.

The tenth paper "Towards a High Performance Computing scalable implementation of Cyber Physical Systems", by Nuno Lopes, Goran Putnik, Luís Ferreira and Bruno Costa, presents the emergent context of Cyber-Physical Systems (CPS) that Industry 4.0 is bringing about, where huge amounts of data are expected and the capacity to process it and (re)act accordingly is required. The scalability and dynamic reconfiguration of process handlers (resources, machines, time, decisions, etc.) are expected to timely respond to the context variants. The CPS intends to support the increasing need to have the physical and digital world connected, but the required computation for handling that, cannot be fitted by increasing the processing capacity of existing resources only. Instead, the authors propose the application of HighPerformance Computing to support the scalable architecture based on more computers, considered the invariant for Cyber-Physical Production Systems (CPPS) implementation, towards a real-time problem solution achievement.

Next paper, the eleventh, "An Industry 4.0 Oriented Tool for Supporting Dynamic Selection of Dispatching Rules based on Kano Model Satisfaction Scheduling”, by Luis Ferreirinha, Sara Baptista, Ângela Pereira, A.S. Santos, J. Bastos, A. M. Madureira and M. L. R. Varela, describes a proposal for a prototype of an I4.0 oriented decision support tool for dynamic production scheduling, a common resources optimizing problem. On a single-machine production scheduling environment, the scheduling tasks are selected and reordered through constructive heuristics, towards an autonomous and dynamic scheduling, that better fits the degrees of satisfaction of the Kano's Model. The prototype was tested on dynamic problems with stochastic characteristics.

The twelfth paper, "Real-Time System for Automatic Cold Strip Surface Defect Detection”, by Pavel Kostenetskiy, Rustem Alkapov, Nikita Vetoshkin, Roman Chulkevich, Ilya Napolskikh and Ostap Poponin, describes the development of a prototype system for real-time automatic cold strip surface defect detection and classification on rolled metal, considered essential for quality assurance in metallurgic industry. According to the authors, the traditional human visionbased method of defects detection is not appropriate for Industry 4.0 requirements, since the rolling rate cannot be so high, and the classification of defects is difficult to obtain. The system architecture, their components and its implementation are documented, and the detection and classification processes are detailed. The proposed system recognizes defects using special video cameras for image capture and the vison library OpenCV for image processing, and a machine learning process supported by convolutional neural networks, was explored to train the defects classification, towards the automatization. A windows graphical user interface for managing the system and a RESTful API for interaction between all system components, represents the remain components of the system.

The thirteenth paper, "A real-time optimization algorithm for the integrated planning and scheduling problem towards the context of industry 4.0", by Mário Leite, Telmo Pinto and Cláudio Alves, proposes a new approach based on a metaheuristic real-time algorithm, to integrate Planning and Scheduling of production system, on the context of Industry 4.0 (I40). The authors made an interesting confrontation of these types of problem in the increasing virtual world that requires quality, flexibility, variety and short time of opportunities, that I4.0 promotes, as well as a clear explanation of the variable neighborhood descent algorithm used on its approach. The computational experiments and obtained results are described too.

The fourteenth paper "Applying Methods of Machine Learning in the Task of Intrusion Detection Based on the Analysis of Industrial Process State and ICS Networking”, by Alexander N. Sokolov, Ilya A. Pyatnitsky and Sergei K. Alabugin, exposes, for sure, one of the most critical subjects on Industry 4.0 (I40), the Security. Cyberattacks have a broad platform for growth in the I40 as global digitization and connection infra-structure, that threatens now the no more isolated ICS of industrial facilities. After demonstrated that traditional protection mechanisms do not fit I40 threats, the authors propose a new approach for attacks detection based on supervised machine learning 
algorithms to classify attacks and recurrent neural networks to detect intrusions. The experiments for training and validations were made on top of industrial datasets and, according to the obtained results, high efficiency and accuracy in detection were achieved, compared with existing methods and algorithms. This is another paper of the SIS that emphasizes the role played by artificial intelligence in I4.0 scenarios.

The fifteenth paper, "Development of an Anthropomorphic Mobile Manipulator with Human, Machine and Environment Interaction", by Gonçalves, Fernando, Ribeiro, Tiago, Garcia, Inês,Ribeiro, Fernando A, Monteiro, Caetano and Lopes, Gil, describes the work-in-process of a manipulator robot that intends to act in real-time using neural networks over acquired sensorial information. Following an anthropomorphic model, the robot interacts using arms and interpreting sounds.

The sixteenth paper, "Machine learning based manufacturing control system for intelligent cyberphysical systems", by Vaibhav Shah and Goran D. Putnik, emphasizes the subject explored in the paper one, where Cyber-Physical Systems (CPS) frameworks and definitions are not correctly understood. The authors propose an architecture that allows the control's supporting system reprogramming, for manufacturing process reconfiguration. Based on machine-learning algorithms, the work points the path towards an Intelligent CPS.

The seventeenth paper, "How Industry 4.0 Can Enhance Lean Practices”, by Ana C. Pereira, José Dinis-Carvalho, Anabela C. Alves and Pedro Arezes, presents a systematic literature review for the impact of Industry 4.0 (I40) on Lean Practices of the adhering organizations. According to the authors, although the integration between I4.0 and Lean Production is already studied and documented, the implementation of the arising new technologies in existing lean practices, needs study and research.

The eighteenth paper, "Human factor in industry of the future - knowledge acquisition and motivation", by Dorota Stadnicka, Paweł Litwin and Dario Antonelli, explores one of the most critical domains in future industries, the role played by the human. The Industry 4.0 strengthens machines and humans' collaborating, and human dependency decreasing (seventh paper). The authors expose methods for human cognitive capacity alignment and motivation increasing, for better preparing future industry workers.

At last, the nineteenth paper, "Industry 4.0 and Industrial Revolutions: an Assessment based on Complexity" by Pedro Pinheiro, Goran D. Putnik, Alrenice Castro, Hélio Castro, Rodrigo Dal Bosco Fontana and Fernando Romero, represents an interesting perspective of the on-going Industry 4.0, announced as the fourth industrial revolution. The authors explore the impacts of this event on existing systems, considering paradigms, persons and technologies. An interesting question is analyzed: " $(\ldots)$ the fourth industrial revolution is effectively underway, or is it still only a vision of the future (...)". Obtained curious results as an interesting closing way for this SI.

Finally, it is expected that this SI can contribute to: i) the improvement of understanding the Industry 4.0 and Manufacturing emergent context; ii) disclose the more relevant research and developments in I4.0 and related science and technology; iii) awareness level of the scientific and industry communities for the need to be aligned to better fit the requirements and explore the potential of I4.0 and iv) enlighten the relevance of an effective human-machine collaboration, the essential condition for the expected symbiose of both in future industries.

\section{ACKNOWLEDGMENT}

First, our acknowledgments and greatest thanks go to Professor Bosko Rasuo, Editor-in-Chief of the FME Transactions, for his highest support and professionalism and, more importantly, his highest collaboration, understanding and patience during the development of this Special Issue. Next, our acknowledgments go to the authors, for their contributions and collaboration and especially to the authors who also served as reviewers, and other reviewers, for their great effort during the review process and for the suggestions they provided to the authors. Acknowledgments go also to our institutions Univeristy of Minho and Polytechnic Institute of Cávado and Ave, and to the Research centres within which this project on this Special Issue has been developed, namelly to ALGORITMI Research Center of the University of Minho and 2Ai - Applied Artificial Intelligence Laboratory Research Center of Polytechnic Institute of Cavado and Ave.

The guest editors want to acknowledge that this work has been supported by FCT - Fundação para a Ciência e Tecnologia, Portugal, within the Project Scope: UID/CEC/00319/2019

Guest Editors:

Goran D. Putnik

Professor Department of Production and Systems Engineering Algoritmi Research Centre University of Minho, Portugal E-mail address: putnikgd@dps.uminho.pt

Luis Gonzaga Martins Ferreira

Assistant Professor School of Technology Polytechnic Institute of Cávado and Ave, Portugal Algoritmi Research Centre, University of Minho, Portugal E-mail address: lufer@ipca.pt

$10^{\text {th }}$ July, 2019 